\title{
Comparison of sirtuin 1 level and related blood factors in diabetic and healthy subjects
}

Porównanie stężenia sirtuiny 1 i parametrów zależnych we krwi osób chorych na cukrzycę i zdrowych

\author{
${ }^{1}$ Mehrali Rahimi, ${ }^{2}$ Somayeh Ghanbari, ${ }^{3}$ Mozafar Khazaei, ${ }^{2}$ Elham Niroman
}

${ }^{1}$ Department of Internal Medicine, Associate Professor of Endocrinology and Metabolism, Kermanshah University of Medical Sciences, Kermanshah, Iran

${ }^{2}$ Department of Internal Medicine, Kermanshah University of Medical Sciences, Kermanshah, Iran

${ }^{3}$ Fertility and Infertility Research Center, Health Technology Institute, Fertility and Infertility Research Center, Health Technology Institute, Kermanshah University of Medical Sciences, Kermanshah, Iran

\footnotetext{
Abstract

Introduction: Diabetes (II) is a chronic metabolic disease with many side effects. Sirtuin 1 (SIRT1) regulates prominent cellular processes such as apoptosis, aging, and metabolism of the cell, and it seems to play an important role in type 2 diabetes.

The aim of the study was to compare the serum level of SIRT1 and related biochemical factors in patients with controlled and uncontrolled diabetes with healthy subjects.

Methods: Type 2 diabetic patients were randomly divided into controlled and uncontrolled fasting blood glucose (FBG) and healthy individuals as the control group ( $n=50$ /group). Serum levels of SIRT1, haemoglobin $\mathrm{A}_{1 \mathrm{C}}\left(\mathrm{HbA}_{1 \mathrm{c}}\right.$ ), low-density lipoprotein (LDL), highdensity lipoprotein (HDL), triglyceride (TG), FBG, and body mass index (BMI) were evaluated.

Results: The mean \pm SD level of SIRT1 in the uncontrolled diabetic group $(3.96 \pm 2.52)$ was significantly $(p<0.001)$ lower than that of the controlled diabetic $(9.22 \pm 4.11)$ and healthy subjects $(10.65 \pm 2.2)$. The levels of $\mathrm{HbA}_{1 c^{\prime}}, \mathrm{FBG}, \mathrm{LDL}, \mathrm{HDL}$, and TG indicated significant differences between the groups $(p<0.05)$. There was a significant reverse correlation between SIRT1 with HbA ${ }_{1 c^{\prime}}$ FBG, age, and BMI $(p<0.05)$ and a positive significant correlation between BMI and $\operatorname{HbA}_{1 \mathrm{c}}(p=0.007)$.

Conclusions: Sirtuin 1 is associated with improving glucose homeostasis. Therefore, it can be considered as a new therapeutic target for prevention and treatment of type 2 diabetes.

Key words:

diabetes mellitus, sirtuin 1 , haemoglobin $\mathrm{A}_{1 \mathrm{c}^{\prime}}$ FBG.
} 


\section{Introduction}

Diabetes mellitus is one of the most serious health problems, with a prevalence of $5.1 \%$ to $5.8 \%$ in Iran [1], and it is associated with various disorders that lead to destruction and impaired function of organs. Type 2 diabetes is a heritable condition, with $90 \%$ of affected children and youths having a firstor second-degree relative who also has type 2 diabetes [2, 3]. $\mathrm{HbA}_{1 \mathrm{c}}$, as a convenient biomarker in the assessment of diabetes, is associated with accurate regulation of blood glucose. It accounts for about $5 \%$ of total haemoglobin in healthy subjects and increases to 2-3-fold in patients with diabetes. $\mathrm{HbA}_{1 \mathrm{c}}$ (a glycated protein) was considered as an indicator for control of long-term glycaemia, and enhanced serum level of $\mathrm{HbA}_{1 \mathrm{c}}$ $(\geq 6.5 \%)$ is indicative of irregular blood glucose status [4].

The family of sirtuin (SIRT) proteins with $\mathrm{NAD}^{+}$-dependent deacetylase activity was first isolated from yeast. These proteins regulate various biological processes from DNA repair and genome stability to lipid and glucose homeostasis [5]. SIRTs are the key regulators of cell survival and the lifespan of the organism [6]. Seven sirtuins have been found in the mammalian body, which are located within different parts of the cell and have a variety of functions. Over the past 10 years, an explosion has occurred in the therapeutic potential of SIRT activation, particularly SIRT1 and SIRT3, in several diseases. However, there is still little information about the function of these proteins in the body [7]

Among sirtuins, SIRT1 is more widely considered and has a diverse biological function in various organs of the body, which includes elevated insulin sensitivity, regulation of cholesterol and lipid, and circadian rhythms [8]. SIRT1 is found in the brain, liver, pancreas, adipose tissue, muscle, and heart [9]. Studies have shown the association of SIRT in the pathogenesis of several diseases, namely: SIRT1 has tumour suppressor activity in cancer and age-related disorders [7]. The expression of SIRT1 increases by caloric restriction [10]. SIRT1 also regulates insulin secretion of pancreatic $\beta$ cells and protects them from oxidative stress and inflammation. SIRT1 plays a positive role in insulin signalling in adipocyte and muscle cells, as well as improving aerobic metabolism [11].

The expression of SIRT1 gene was reduced in obese children and revealed significant negative correlations with waist circumference, body mass index (BMI), and insulin resistance (IR). SIRT1 targeting can be valuable in preventing obesity-related IR in childhood and adolescence [12].

Mounting evidence indicates that SIRT1 suppression promotes systemic inflammation, increases oxidative stress, and reduces aerobic metabolism, while its induction is associated with improved insulin sensitivity and glomerular haemostasis [13]. Because SIRT1 is able to regulate important cellular processes, it could be seen as a promising therapeutic target for type 2 diabetes.

The aim of the study was to compare the serum levels of SIRT1 and related biochemical factors as well as BMI in patients with controlled and patients with uncontrolled diabetes and healthy subjects.

\section{Material and methods}

In this descriptive-analytical study, patients with diabetes (30-50 years old), who had been referred to a diabetes clinic, were selected based on the criteria of the study. This study was approved by the Ethics Committee of our University (CODE No. IR.KUMS.REC.1396.297), and informed consent was signed by the participants. The subjects were divided into three groups ( $n=50 /$ group), including: patients with controlled diabetes $\left(\mathrm{HbA}_{1 \mathrm{c}} \leq 6.5\right)$, patients with uncontrolled diabetes $\left(\mathrm{HbA}_{1 \mathrm{c}} \geq 6.5\right)$, and healthy participants (3). The routine diabetes treatment was insulin therapy in the clinic.

The exclusion criteria were as follows: age < 30 or $>50$ years; duration of insulin-dependent diabetes less than three years; symptoms or clinical evidence of ischaemic heart disease; taking lipid-lowering drugs; severe renal disease; proliferative retinopathy; thyroid disease; malignant tumours; and alcoholism. The study criteria were as follows: at least three years of history of type 2 diabetes, FBG more than $126 \mathrm{mg} / \mathrm{d}$, no use of lipidlowering drugs in the six months prior to study, and body mass index (BMI) less than 40 [14].

Blood samples were collected in standard conditions after 10-12 hours of fasting. Sera were separated and kept at $-20^{\circ} \mathrm{C}$. Serum levels of SIRT1 were measured using the ELISA method (US Biovision kit). FBG, $\mathrm{HbA}_{1 \mathrm{c}}$ and LDL, HDL, triglyceride, and cholesterol levels were measured enzymatically using commercial assay kits (Randox laboratories Ltd., United Kingdom).

\section{Data analysis}

Data were reported as mean \pm SD and median (interquartile range), and analysis was conducted using Mann-Whitney $U$ test, Kruskal-Wallis, and one-way ANOVA. Correlation analysis was performed using Pearson correlation coefficient, and chi-square was used for analysis of nominal variables. All statistical tests were performed by SPSS software version 20, and a significance level of $p<0.05$ was considered.

\section{Results}

The level of SIRT1 in the uncontrolled diabetic group (3.96 $\pm 2.52)$ was significantly $(p<0.001)$ lower than the controlled diabetic group $(9.22 \pm 4.11)$ and healthy subjects (control group) (10.65 \pm 2.2 ) (Fig. 1). BMI was also significantly different between groups ( $p<0.001)$, and in the healthy subjects $(24.97$ $\pm 4.68)$ it was less than in the controlled $(28.52 \pm 3.97)$ and uncontrolled $(28.98 \pm 2.91)$ diabetic groups. There was a significant difference in $\mathrm{HbA}_{1 \mathrm{c}}$ between groups; the control group (4.8 [4-5.2]) had the lowest median and the uncontrolled diabetic group (9.45 [8-14.2]) had the highest level.

There was a significant difference in LDL in the control group (92.5 [56-170]) compared to the uncontrolled diabetic group (95 [43-190]) and the controlled diabetic group (76 [48-139]) $(p<0.05)$ and TG in the control group (182.5 [65-324]) compared to the uncontrolled diabetic group (145.5 [57-350]) and the controlled diabetic group (120.5 [37-274]) $(p<0.001)$, and 
there was no significant difference in the HDL between groups $(p>0.1)$ (Table I). A comparison of the correlation of different variables showed that there was significant reverse correlation between SIRT1 and $\mathrm{HbA}_{10}, \mathrm{FBG}$, age, and BMI $(p<0.05)$. Also, there was a significant positive correlation between $\mathrm{BMl}$ and $\mathrm{HbA}_{1 \mathrm{c}}(p=0.007)$ (Table II).

\section{Discussion}

In this study SIRT1, $\mathrm{HbA}_{1 \mathrm{c}}$, and related biochemical factors of serum, and BMI were compared in patients with controlled and uncontrolled diabetes and healthy subjects. Patients with uncontrolled diabetes had the lowest serum level of SIRT1 and the highest levels of $\mathrm{HbA}_{1 \mathrm{c}}$. The most important findings in the variable correlation were a significant and reverse relationship of SIRT1 with $\mathrm{HbA}_{1 \mathrm{c}}$ and $\mathrm{FBG}$, and a direct significant correlation between $\mathrm{BMI}$ and $\mathrm{HbA}_{1 \mathrm{c}}$. In addition, our results showed that serum levels of SIRT1 were higher in patients with controlled

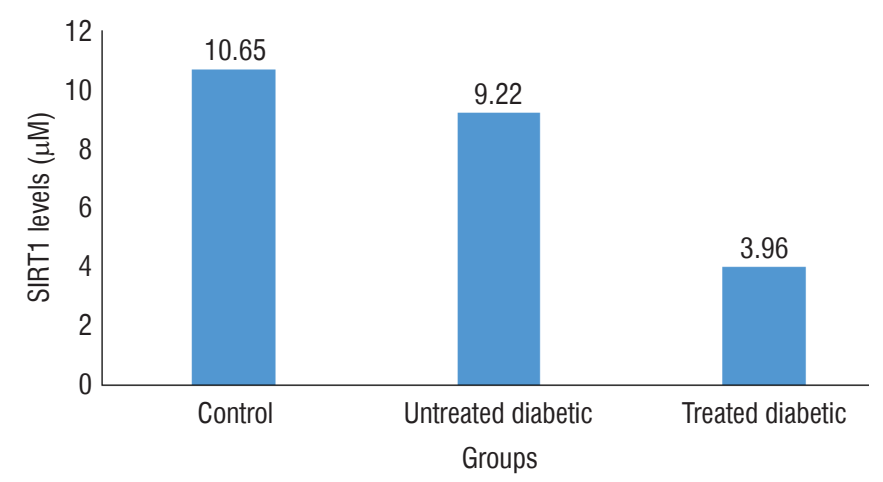

Data show the mean $\pm \operatorname{SD}(n=50)$ : control group $(10.65 \pm 2.2)$, controlled diabetic $(9.22 \pm 4.11)$ and uncontrolled diabetic $(3.96 \pm 2.52)$ groups; a - significant difference $(p<0.05)$ with control group

Figure 1. Serum levels of SIRT 1 in the control and experimental groups

Table I. Median (IQR) and mean \pm SD of variables in uncontrolled and controlled diabetic and healthy subjects

\begin{tabular}{lllll}
\hline Characteristics & Healthy & Controlled diabetes & Uncontrolled diabetes & $p$ value \\
\hline FBG & $89.50(77-99)^{\mathrm{a}}$ & $108(70-178)^{\mathrm{b}}$ & $158(137-307)^{\mathrm{c}}$ & $<0.001 \dagger$ \\
\hline $\mathrm{HbA}_{1 \mathrm{c}}(\%)$ & $4.80(4-5.2)^{\mathrm{a}}$ & $6.50(5.4-7.02)^{\mathrm{b}}$ & $9.45(8-14.2)^{\mathrm{c}}$ & $<0.001 \dagger$ \\
\hline $\mathrm{BMI}$ & $24.97 \pm 4.68^{\mathrm{a}}$ & $28.52 \pm 3.97^{\mathrm{b}}$ & $28.98 \pm 2.91^{\mathrm{b}}$ & $<0.001 \ddagger$ \\
\hline $\mathrm{TG}$ & $182.50(65-324)^{\mathrm{b}}$ & $120.50(37-274)^{\mathrm{a}}$ & $145.50(57-350)^{\mathrm{ab}}$ & $<0.001 \dagger$ \\
\hline $\mathrm{LDL}$ & $92.50(56-170)^{\mathrm{b}}$ & $76(48-139)^{\mathrm{a}}$ & $95(43-190)^{\mathrm{ab}}$ & $0.015 \dagger$ \\
\hline $\mathrm{HDL}$ & $38.74 \pm 7.45$ & $42.04 \pm 8.39$ & $41.62 \pm 7.06$ & $0.067 \ddagger$ \\
\hline
\end{tabular}

Median (interquartile range) and mean [standard deviation] are presented for data without and with normality test, respectively. Means or medians with same superscript letters are not significantly different $(p>0.05)$. ‡ One-way ANOVA test followed by Tukey's test was used. $†$ KruskalWallis test followed by Dunn's multiple comparisons test was used

FBG - fasting blood glucose; SIRT1 - sirtuins 1; $\mathrm{HbA}_{1 \mathrm{c}}$ - haemoglobin $\mathrm{A}_{1 \mathrm{c}}$; $\mathrm{BMI}$ - body mass index; TG - triglycerides; LDL - low-density lipoprotein; HDL - high-density lipoprotein.

Table II. Correlation of SIRT1 with FBG and $\mathrm{HbA}_{1 \mathrm{c}}$

\begin{tabular}{lllll}
\hline SIRT1 & $\mathrm{HbA}_{1 \mathrm{c}}$ & $\mathrm{FBG}$ & Age & $\mathrm{BMl}$ \\
\hline Pearson correlation & -0.373 & -0.295 & -0.175 & -0.324 \\
\hline$p$ value & $<0.001$ & $<0.001$ & 0.032 & $<0.001$ \\
\hline$n$ & 150 & 150 & 150 & 150 \\
\hline
\end{tabular}

SIRT1 - sirtuin 1; $\mathrm{HbA}_{1 \mathrm{c}}$ - haemoglobin $\mathrm{A}_{1 \mathrm{c}} ; \mathrm{FBG}$ - fasting blood glucose; $\mathrm{BMI}$ - body mass index 
compared to patients with uncontrolled diabetes, which is in line with other studies $[9,15]$.

Kitada et al. showed that SIRT1 promotes anti-diabetic effects by modulating insulin secretion, improving insulin resistance, and regulating circadian rhythms [9]. Yacoub et al. implied a lower expression of SIRT1 in oxidative stress and chronic metabolic stress [16]. In agreement with their studies, we documented a significant, lower level of SIRT1 in patients with uncontrolled diabetes, and these findings can be attributed to the fact that hyperglycaemia leads to oxidative stress and ROS generation [17].

Additionally, SIRT1 protects $\beta$-cells against different oxidative stress by suppressing NF- $\kappa \beta$ signalling pathway. In $\beta$-cellspecific SIRT1 over-expression (BESTO) mice, higher SIRT1 levels improve glucose tolerance and increase insulin secretion $[18,19]$. Furthermore, SIRT1 over-expression increases ATP formation via repression of uncoupling protein 2 (UCP2) in $\beta$-cells $[19,20]$ that cause $\mathrm{Ca}^{2+}$-dependent insulin exocytosis [21]. SIRT1 also elevates the transcription and activation of Maf $A$ and Neuro D, maintaining insulin production and enhancing $\beta$-cell survival in vivo. It modulates insulin secretion from $\beta$-cells through UCP2, FOXO1, and NAD + metabolism, leading to protective effects on different toxic stresses [22]. Also, Calabrese et al. reported that the expression of SIRT1 and SIRT3 was significantly lower in patients with uncontrolled diabetes [23].

Our results are in agreement with previous studies [23, 24] in which mean BMI was significantly higher in uncontrolled diabetic groups. Obesity is a risk factor for diabetes. There was no significant difference in the serum levels of HDL between groups. However, the serum levels of TG and LDL were higher in the control group. SIRT1 deacetylates the nuclear histones, indicating its repressive effect in gene transcription [25]. Furthermore, the metabolic activity of SIRT1 depends on the deacetylation of non-histone proteins such as peroxisome proliferator-activated receptor (PPAR)- $\gamma$ and $\alpha$, coactivator- $1 \alpha$ (PGC- $1 \alpha$ ), insulin receptor substrate 2, and mitochondrial UCP-2. Due to its deacetylation potential, SIRT1 regulates insulin secretion, myogenesis, and adipogenesis [26].

Our study also shows that serum levels of SIRT1 significantly decreased in patients with uncontrolled diabetes with high levels of $F B G$ and $\mathrm{HbA}_{1 \mathrm{c}}$. SIRT1 plays an important role

\section{References}

1. Azimi-Nezhad M, Ghayour-Mobarhan M, Parizadeh M, et al. Prevalence of type 2 diabetes mellitus in Iran and its relationship with gender, urbanisation, education, marital status and occupation. Singapore Med J 2008; 49: 571-576.

2. Fuchsberger C, Flannick J, Teslovich TM, et al. The genetic architecture of type 2 diabetes. Nature 2016; 536: 41-47. doi: 10.1038/ nature18642

3. Copeland KC, Zeitler P, Geffner M, et al. Characteristics of adolescents and youth with recent-onset type 2 diabetes: the TODAY in controlling glucose homeostasis. Indeed, under diabetic conditions, the activity of the SIRT1 protein declines in various tissues. SIRT1 is a regulator of the metabolic response of mammalians to caloric restriction [27]. A report by Khowailed et al. indicated that a significant increase in SIRT1 protein in the pre-diabetes caloric restriction group was significantly related to the decrease in the serum levels of glucose [28].

In humans, a number of single nucleotide polymorphisms (SNPs) associated with diabetes and obesity have been recognised in the SIRT1 genes, suggesting that SIRTs may play a central role in the development of these conditions [29]. The fact that the level of SIRT1 is reduced with high-fat feeding and aging indicates that decreased SIRT1 activity is related to metabolic abnormalities consistent with diabetes and SNPs that result in reduced SIRT activity related to the development of the metabolic syndrome. Moreover, it has been suggested that SIRT activity may be a mechanistic link between over-nutrition, aging, and diabetes. Accordingly, STIR activators seem to be an effective therapeutic pipeline in diabetes and its complications [15] .

SIRT1 activation is associated with improved glucose homeostasis. The interplay with other different variables in controlled and uncontrolled diabetes could indicate the critical role of SIRT1 in the pathogenesis of diabetes. The present study has shown that the serum level of SIRT1 in patients with diabetes reduced with increasing age, but it is not surprising that different results among diabetic patients could be related to the rate of obesity, treatment regimens, age, and sex [30]. Our study had some limitations such as small sample size, assessing just one type of sirtuin, and lack of gender consideration. Hence, there is a need for a complementary study with large sample size and a study of same protocols in obese children with and without diabetes.

\section{Conclusions}

SITR1 is associated with improved glucose homeostasis, and the serum level of SIRT1 in the patient with diabetes was reduced; therefore, it can be considered as a new therapeutic target for prevention and treatment of type 2 diabetes.

cohort at baseline. J Clin Endocrinol Metab 2011; 96: 159-167. doi: 10.1210/jc.2010-1642

4. Yazdanpanah S, Rabiee M, Tahriri M, et al. Evaluation of glycated albumin (GA) and GA/HbA1c ratio for diagnosis of diabetes and glycemic control: a comprehensive review. Crit Rev Clin Lab Sci 2017; 54: 219-232. doi: 10.1080/10408363.2017.1299684

5. Donmez G, Guarente L. Aging and disease: connections to sirtuins. Aging Cell 2010; 9: 285-290. doi: 10.1111/j.1474-9726.2010.00548.x

6. Gillum MP, Kotas ME, Erion DM, et al. SirT1 regulates adipose tissue inflammation. Diabetes 2011; 60: 3235-3245. doi: 10.2337/ $\mathrm{db} 11-0616$ 
7. Delevatti RS, Pinho CDF, Kanitz AC, et al. Glycemic reductions following water-and land-based exercise in patients with type 2 diabetes mellitus. Complement Ther Clin Pract 2016; 24: 73-77. doi: 10.1016/j.ctcp.2016.05.008

8. Banks AS, Kon N, Knight C, et al. SirT1 gain of function increases energy efficiency and prevents diabetes in mice. Cell Metab 2008; 8: 333-341. doi: 10.1016/j.cmet.2008.08.014

9. Kitada M, Koya D. SIRT1 in type 2 diabetes: mechanisms and therapeutic potential. Diabetes Metab J 2013; 37: 315-325. doi: 10.4093/dmj.2013.37.5.315

10. Schug TT, Li X. Sirtuin 1 in lipid metabolism and obesity. Ann Med 2011; 43: 198-211. doi: 10.3109/07853890.2010.547211

11. Zhou M, Luo J, Zhang $\mathrm{H}$. Role of Sirtuin 1 in the pathogenesis of ocular disease. Int J Mol Med 2018; 42: 13-20. doi: 10.3892/ ijmm.2018.3623

12. Sadeghabadi ZA, Nourbakhsh M, Pasalar P, et al. Reduced gene expression of sirtuins and active AMPK levels in children and adolescents with obesity and insulin resistance. Obesity research Clin Pract 2018; 12: 167-173. doi: 10.1016/j.orcp.2017.10.004

13. Liang F, Kume S, Koya D. SIRT1 and insulin resistance. Nat Rev Endocrinol 2009; 5: 367-373. doi: 10.1038/nrendo.2009.101.

14. American Diabetes Association. Diagnosis and classification of diabetes mellitus. Diabetes Care 2010; 33 (Suppl 1): S62-S69.

15. Huynh FK, Hershberger KA, Hirschey MD. Targeting sirtuins for the treatment of diabetes. Diabetes Manag (Lond) 2013; 3: 245-257. doi: $10.2217 / \mathrm{dmt} .13 .6$

16. Yacoub R, Lee K, He JC. The role of SIRT1 in diabetic kidney disease. Front Endocrinol 2014; 5: 166. doi: 10.3389/fendo.2014.00166

17. Noh H, King G. The role of protein kinase $\mathrm{C}$ activation in diabetic nephropathy. Kidney Int Suppl 2007; 72: S49-S53. doi: 10.1038/ sj.ki.5002386

18. Liu H-W, Chang S-J. Moderate exercise suppresses NF-кB signaling and activates the SIRT1-AMPK-PGC1a axis to attenuate muscle loss in diabetic db/db mice. Front Physiol 2018; 9: 636. doi: 10.3389/fphys.2018.00636

19. Cao Y, Jiang $X, M a H$, et al. SIRT1 and insulin resistance. J Diabetes Complicat. 2016; 30: 178-183. doi: 10.1016/j.jdiacomp.2015.08.022
20. Schwer B, Verdin E. Conserved metabolic regulatory functions of sirtuins. Cell Metab 2008; 7: 104-112. doi: 10.1016/j.cmet.2007.11.006

21. Turkmen K, Karagoz A, Kucuk A. Sirtuins as novel players in the pathogenesis of diabetes mellitus. World J Diabetes 2014; 5: 894900. doi: 10.4239/wjd.v5.i6.894

22. Chalkiadaki A, Guarente L. Sirtuins mediate mammalian metabolic responses to nutrient availability. Nat Rev Endocrinol 2012; 8: 287 296. doi: $10.1038 /$ nrendo.2011.225

23. Calabrese V, Cornelius C, Leso V, et al. Oxidative stress, glutathione status, sirtuin and cellular stress response in type 2 diabetes. Biochim Biophys Acta Mol Basis Dis 2012; 1822: 729-736. doi: 10.1016/j.bbadis.2011.12.003

24. Saremi A, Shahrjerdi S, Kavyani A. The Effect of Aerobic Training on Metabolic Parameters and Serum Level of Sirtuin1 in Women with Type 2 Diabetes. AMUJ 2016; 19: 88-97.

25. Bagul PK, Deepthi N, Sultana R, Banerjee SK. Resveratrol ameliorates cardiac oxidative stress in diabetes through deacetylation of NFkB-p65 and histone 3. J Nutr Biochem 2015; 26: 1298-1307. doi: 10.1016/j.jnutbio.2015.06.006

26. Ye X, Li M, Hou T, et al. Sirtuins in glucose and lipid metabolism. Oncotarget 2017; 8: 1845-1859. doi: 10.18632/oncotarget.12157

27. Chen D, Guarente L. SIR2: a potential target for calorie restriction mimetics. Trends Mol Med 2007; 13: 64-71. doi: 10.1016/j.molmed. 2006.12.004

28. Khowailed EA, Rashed LA, Ahmed Seddiek H, et al. Is the effect of caloric restriction on type 2 diabetes mellitus in rats mediated via sirtuin-1. Med J Cairo Univ 2015; 83: 357-365.

29. Shimoyama Y, Suzuki K, Hamajima N, Niwa T. Sirtuin 1 gene polymorphisms are associated with body fat and blood pressure in Japanese. TransI Res 2011; 157: 339-347. doi: 10.1016/j.trsl.2011.02.004

30. de Kreutzenberg SV, Ceolotto G, Papparella I, et al. Downregulation of the longevity-associated protein sirtuin 1 in insulin resistance and metabolic syndrome: potential biochemical mechanisms. Diabetes 2010; 59: 1006-1015. doi: 10.2337/db09-1187 